\title{
Progress toward development of a proteostasis drug for myocilin-associated glaucoma
}

\author{
Dustin JE Huard ${ }^{1}$ \& Raquel L Lieberman*,1 \\ ${ }^{1}$ School of Chemistry \& Biochemistry, Georgia Institute of Technology, Atlanta, GA 30332-0400, USA \\ *Author for correspondence: raquel.lieberman@chemistry.gatech.edu
}

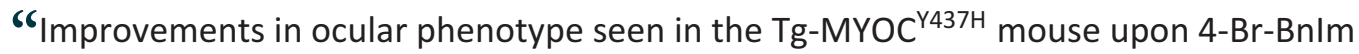
treatment [18] pave the way for development of more potent molecules [12] that could be translated to the clinic. Not only would such a therapeutic serve as the first disease-modifying drug for glaucoma, it would realize the promise of Hsp90s as a drug target for neurodegenerative diseases [20]."

First draft submitted: 9 March 2018; Accepted for publication: 13 March 2018; Published online: 23 May 2018

Keywords: aggregation $\bullet$ glaucoma $\bullet$ Grp94 $\bullet$ inhibitor $\bullet$ meshwork $\bullet$ myocilin $\bullet$ proteostasis $\bullet$ trabecular

Glaucoma, a collection of neurodegenerative diseases associated with loss of optic nerve function, is a leading cause of blindness worldwide, affecting upward of 60 million individuals, including approximately 3 million people with mutations in the gene encoding for myocilin [1]. Despite its prevalence, there are currently no available treatments that directly address molecular causes of glaucoma. Current therapeutic treatments focus on alleviating elevated intraocular pressure (IOP), the as-yet only known and clinically-addressable risk factor for glaucoma. An imbalance between the production and outflow of aqueous humor $(\mathrm{AH})$, the fluid secreted by the ciliary body that provides nutrients to the avascular anterior eye, tracks with IOP elevation. Reduction of IOP is accomplished pharmacologically by modulating $\mathrm{AH}$ production or drainage, or surgically [2].

For individuals harboring mutations in myocilin, restoring proteostasis is a promising direction for drug development. When proteostasis is normal, protein production and protein clearance work in concert to prevent protein accumulation/aggregation. Mutations or other factors can cause protein misfolding, enabling these proteins to readily accumulate and aggregate [3]. While most cells deal with proteostasis problems by undergoing apoptosis, cells that are long-lived, like trabecular meshwork (TM) cells [4], are programmed to avoid cell death [5] and, consequently, can be toxified by the accumulation of misfolded proteins [6]. Mutations that cause degenerative diseases often accelerate the propensity of proteins to aggregate [7].

In its wild-type (WT) state, myocilin, a protein of unknown biological function, is secreted to the TM [2], an extracellular matrix (ECM) tissue that regulates $\mathrm{AH}$ outflow in the anterior eye. TM dysfunction thus contributes to glaucoma-associated IOP elevation. Numerous nonsynonymous coding mutations in myocilin, localized predominantly to the C-terminal OLF domain, lead to an autosomal-dominant inherited form of glaucoma [1]. Mutant OLF domains exhibit compromised stability [8], resulting in misfolding and subsequent retention of mutant myocilin within the endoplasmic reticulum (ER) of TM cells [9]. Nonsecretion and accumulation of misfolded mutant myocilin toxifies TM cells, leading to ER stress and eventual cell death. TM cell loss correlates with dysregulation of $\mathrm{AH}$ outflow and increased IOP, facilitating eventual vision loss [1]. Individuals harboring distinct truncated forms of myocilin protein, and myocilin knock-out mice, do not develop glaucoma [1], so glaucoma caused by mutant myocilin is due to a toxic gain of function, namely, aggregation.

Mutant myocilin fails to be removed by the ER-associated degradation (ERAD) molecular chaperone system, resulting in its toxic accumulation. Grp94, the ER-resident Hsp90 molecular chaperone and key ERAD player [10], attempts to triage mutant myocilin but is not successful [9]. Grp94 co-aggregates with mutant myocilin and the two proteins are retained in the ER [9,11]. Aberrant co-aggregation is recapitulated in vitro: not only does Grp94 
co-aggregate with stability compromised OLF, but it enhances the rate at which aggregates form [11,12]. Of note, aggregates of mutant myocilin exhibit characteristics of amyloid in vitro and in cells [13], similar to other proteostasis disorders like Alzheimer's disease.

\section{Strategies to restore proteostasis}

A proteostasis drug to mitigate the toxic gain of functions of mutant myocilin, namely, retention of mutant myocilin in the ER and/or recruitment of Grp94 to mutant myocilin aggregates, could be developed utilizing a variety of strategies. One option is simply to reduce ER stress by administering 4-phenylbutyrate (4PBA) [14]. The targets of 4PBA remain unclear, but myocilin is not one [15]. The lack of a clear molecular target for 4PBA is a disadvantage for structure-activity relationship studies to generate more potent and selective molecules, however. A second approach is to rescue mutant myocilin secretion by stabilizing it against aggregation and enabling it to escape detection by Grp94 [16]. Such a pharmacological chaperone would either provide secreted mutant myocilin the opportunity to perform its biological role or enable it to be engulfed by phagocytosis [17], a major function of TM cells for ECM maintenance, and then degraded by autophagy. Finally, clearance of toxic myocilin aggregates should be possible by disruption of the aberrant interaction between Grp94 and mutant myocilin. Grp94 is essential only during early organismal development [10], the lack of myocilin does not cause glaucoma [1] and knockdown of Grp94 facilitates mutant myocilin clearance [9].

\section{Recent work: inhibiting Grp94}

Recently, the strategy of inhibiting Grp94 to treat myocilin-glaucoma has gained traction. Grp94 has been identified as a target, its molecular mechanism with mutant myocilin has been probed and inhibitors that facilitate mutant myocilin degradation have been developed. The pilot inhibitor, 4-Br-BnIm, is tailored to bind the Grp94 N-terminal nucleotide binding site in preference to that of other Hsp90 paralogs $[11,18]$. Grp94 contains a unique secondary binding pocket within the $\mathrm{N}$-terminal nucleotide binding site due to a 5-amino acid insertion ( ${ }_{182} \mathrm{EDGQ}$ ), enabling it to be selectively inhibited [19].

In initial studies, treatment of HEK cells stably over-expressing I477N mutant myocilin or those transiently overexpressing a variety of other mutants (including W286R, P370L, Y437H, I477N and N480K) with 4-BrBnIm resulted in significant clearance of aggregated myocilin protein; similar results were obtained with TM cells expressing $\mathrm{Y} 437 \mathrm{H}$ myocilin [11]. Importantly, inhibition of Grp94 did not evoke the cell stress response, nor did treatment with 4-Br-BnIm affect maturation of Hsp90-dependent clients Akt and Ras in HEK cells, while mutant myocilin-derived cytotoxicity was alleviated in the TM cells [11]. In vitro, 4-Br-BnIm attenuated the rate enhancement observed on OLF aggregation due to Grp94 and rescued the chaperone from co-aggregating with OLF [11], emphasizing that Grp94 inhibition prevents the aberrant interaction with aggregating OLF. The efficacy of ocular 4-Br-BnIm treatment was further demonstrated in mice [18], using a transgenic mouse expressing human $\mathrm{Y} 437 \mathrm{H}$ mutant myocilin, which serves as a model for myocilin-associated glaucoma (Tg-MYOC $\left.{ }^{\mathrm{Y} 437 \mathrm{H}}\right)$ [14] . Whereas Tg-MYOC ${ }^{\mathrm{Y} 437 \mathrm{H}}$ mice exhibit elevated IOP and deficits to retinal ganglion cell (RGC) count and function, those that received topical ocular delivery of 4-Br-BnIm showed WT levels of IOP and restored RGC count and functionality; 4-Br-BnIm itself did not affect IOP [18]. As in the cell-based studies, less aggregated myocilin was present upon treatment with 4-Br-BnIm and the heat shock response was not observed suggesting that pan-Hsp90 inhibition did not occur [18].

\section{Conclusion}

Myocilin remains one of the only known proteins that if targeted with small molecules could slow or even stop glaucoma progression, and a treatment to ameliorate proteostasis defects caused by mutant myocilin using small molecules is on the horizon. Improvements in ocular phenotype seen in the Tg-MYOC ${ }^{\mathrm{Y} 37 \mathrm{H}}$ mouse upon 4Br-BnIm treatment [18] pave the way for development of more potent molecules [12] that could be translated to the clinic. Not only would such a therapeutic serve as the first disease-modifying drug for glaucoma, it would realize the promise of Hsp90s as a drug target for neurodegenerative diseases [20]. Finally, beyond mutant myocilin, remodeling by TM cells requires numerous ECM protein components to be secreted, a process whose efficiency is compromised upon aging. Thus, in the long term, reducing ER stress by selectively inhibiting Grp94 could prove broadly therapeutic for glaucoma. 


\section{Financial \& competing interests disclosure}

This work was funded by NIH R01EY021205. The authors have no other relevant affiliations or financial involvement with any organization or entity with a financial interest in or financial conflict with the subject matter or materials discussed in the manuscript apart from those disclosed.

No writing assistance was utilized in the production of this manuscript.

\section{References}

1. Tamm ER. Myocilin and glaucoma: facts and ideas. Prog. Retin. Eye Res. 21(4), 395-428 (2002).

2. Donegan RK, Lieberman RL. Discovery of molecular therapeutics for glaucoma: challenges, successes, and promising directions. J. Med. Chem. 59(3), 788-809 (2016).

3. Dobson CM. Protein folding and misfolding. Nature 426(6968), 884-890 (2003).

4. Stamer WD, Clark AF. The many faces of the trabecular meshwork cell. Exp. Eye Res. 158, 112-123 (2017).

5. Kole AJ, Annis RP, Deshmukh M. Mature neurons: equipped for survival. Cell Death Dis. 4, e689 (2013).

6. Bucciantini M, Giannoni E, Chiti $\mathrm{F}$ et al. Inherent toxicity of aggregates implies a common mechanism for protein misfolding diseases. Nature 416(6880), 507-511 (2002).

7. Ross CA, Poirier MA. Protein aggregation and neurodegenerative disease. Nat. Med. 10(Suppl.), S10-S17 (2004).

8. Burns JN, Turnage KC, Walker CA, Lieberman RL. The stability of myocilin olfactomedin domain variants provides new insight into glaucoma as a protein misfolding disorder. Biochemistry 50(26), 5824-5833 (2011).

9. Suntharalingam A, Abisambra JF, O’Leary JC 3rd et al. Glucose-regulated protein 94 triage of mutant myocilin through endoplasmic reticulum-associated degradation subverts a more efficient autophagic clearance mechanism. J. Biol. Chem. 287(48), 40661-40669 (2012).

10. Marzec M, Eletto D, Argon Y. GRP94: an HSP90-like protein specialized for protein folding and quality control in the endoplasmic reticulum. Biochim. Biophys. Acta 1823(3), 774-787 (2012).

11. Stothert AR, Suntharalingam A, Huard DJE et al. Exploiting the interaction between Grp94 and aggregated myocilin to treat glaucoma. Hum. Mol. Genet. 23(24), 6470-6480 (2014).

12. Huard DJE, Crowley VM, Du Y et al. Trifunctional high-throughput screen identifies promising scaffold to inhibit Grp94 and treat myocilin-associated glaucoma. ACS Chem. Biol. 13(4) 933-941 (2018).

13. Orwig SD, Perry CW, Kim LY et al. Amyloid fibril formation by the glaucoma-associated olfactomedin domain of myocilin. J. Mol. Biol. 421(2-3), 242-255 (2012).

14. Zode GS, Kuehn MH, Nishimura DY et al. Reduction of ER stress via a chemical chaperone prevents disease phenotypes in a mouse model of primary open angle glaucoma. J. Clin. Invest. 121(9), 3542-3553 (2011).

15. Burns JN, Orwig SD, Harris JL, Watkins JD, Vollrath D, Lieberman RL. Rescue of glaucoma-causing mutant myocilin thermal stability by chemical chaperones. ACS Chem. Biol. 5(5), 477-487 (2010).

16. Orwig SD, Chi PV, Du Y et al. Ligands for glaucoma-associated myocilin discovered by a generic binding assay. ACS Chem. Biol. 9(2), 517-525 (2014).

17. Buller C, Johnson DH, Tschumper RC. Human trabecular meshwork phagocytosis. Observations in an organ culture system. Invest. Ophthalmol. Vis. Sci. 31(10), 2156-2163 (1990).

18. Stothert AR, Suntharalingam A, Tang X et al. Isoform-selective Hsp90 inhibition rescues model of hereditary open-angle glaucoma. Sci. Rep. 7(1), 17951 (2017).

19. Crowley VM, Huard DJE, Lieberman RL, Blagg BSJ. Second generation Grp94-selective inhibitors provide opportunities for the inhibition of metastatic cancer. Chemistry 23(62), 15775-15782 (2017).

20. Pratt WB, Gestwicki JE, Osawa Y, Lieberman AP. Targeting Hsp90/Hsp70-based protein quality control for treatment of adult onset neurodegenerative diseases. Annu. Rev. Pharmacol. Toxicol. 55, 353-371 (2015). 
